\title{
Les occasions de lire au Nord - Cameroun
}

\section{André Carrée}

\section{OpenEdition}

Journals

Édition électronique

URL : http://journals.openedition.org/trema/2208

DOI : $10.4000 /$ trema.2208

ISSN : 2107-0997

\section{Éditeur}

Faculté d'Éducation de l'université de Montpellier

\section{Édition imprimée}

Date de publication : 1 juin 1995

Pagination : 83-88

ISSN : 1167-315X

\section{Référence électronique}

André Carrée, «Les occasions de lire au Nord - Cameroun », Tréma [En ligne], 7| 1995, mis en ligne le 23 septembre 2013, consulté le 19 avril 2019. URL : http://journals.openedition.org/trema/2208 ; DOI : 10.4000/trema.2208

Ce document a été généré automatiquement le 19 avril 2019

Trema 


\title{
Les occasions de lire au Nord - Cameroun
}

\author{
André Carrée
}

1 Dans les provinces septentrionales du Cameroun, la langue de communication est le foulfouldé. Le français est langue officielle (avec l'anglais), langue de l'école et, d'une façon générale, de l'écrit. Un enfant n'a que de rares occasions d'entendre parler français, si ce n'est à la radio, à la télévision et lors de discussions avec des habitants de la partie méridionale. Cette situation explique en partie les difficultés que rencontrent les élèves dans l'acquisition du français. Il convient cependant de s'interroger sur un autre aspect des choses : la possibilité pour les enfants du Nord du Cameroun de voir du français écrit. La pauvreté de leur pratique de l'oral est-elle ou non compensée par une richesse de contacts avec l'écrit? Quelles occasions les élèves apprenant le français ont-ils, en dehors de l'école, de lire dans cette langue?

\section{L'enquête.}

Comme cadre de l'étude, nous avons choisi Bibemi,sous-préfecture d'environ 6000 habitants, située à 60 kilomètres de Garoua. L'activité y est surtout agricole. On y a ouvert, en septembre 1961, un collège qui n'avait, à l'époque de l'enquête, que des classes de $6^{\mathrm{e}}$.

Il s'agissait de voir quelles occasions les élèves avaient de lire en dehors de l'école. Quels textes ont-ils sous les yeux, en ville, chez eux, dans des lieux publics? Quels livres, bandesdessinées, etc. sont disponibles? C'est à ces deux différentes possibilités de lecture que renverront, dans les lignes suivantes, les termes textes et livres.

4 Les manuels et cahiers de cours sont donc exclus de cette enquête. Il convient cependant de signaler que peu d'élèves possèdent tous les manuels de la classe. Le salaire mensuel d'un secrétaire est d'environ $500 \mathrm{~F}$ français, un manuel neuf coûtant entre 30 et $80 \mathrm{~F}$, il n'est pas rare qu'un professeur fasse cours dans une classe où un élève sur 10 possède le manuel. Les textes écrits dans les salles de classe sont également peu nombreux : textes écrits au tableau noir, planning des corvées de balayage. 
5 L'enquête s'est déroulée pendant une semaine de cours de français. Les élèves, par groupes de 2 ou 3, ont rempli des fiches en deux parties. Dans la première, ils ont établi un inventaire des textes lus dans la ville, regroupés par rubriques (vêtements, boissons, automobiles, etc.). Dans la seconde, chaque élève a indiqué s'il avait lu un livre pendant le mois précédent (qui comportait une semaine de vacances), le cas échéant son titre et le lieu où il se l'était procuré.

6 Une difficulté a consisté à rendre les élèves conscients des textes apparaissant dans la ville et à éviter qu'ils n'en inventent (dans l'espoir d'une bonne note).

\section{Les textes}

\subsection{La ville}

7 D'une façon générale, la ville ne semble offrir que de rares occasions de lire. Voici cependant les domaines où les élèves ont trouvé des textes écrits en français.

8 Sur des panneaux ou pancartes. La liste de 20 items semble pratiquement exhaustive. On y lit des expressions comme Hôpital Silence, Gendarmerie de Bibemi, Station zootechnique et vétérinaire de Bibemi, etc.

9 Sur des vêtements. Les élèves ont relevé 36 items. Il s'agit de noms de marques (Adidas sur les chaussures, Lacoste sur les chemises), mais aussi de textes sur les tee-shirts, les pagnes (Journée internationale de la femme, Tout passe mais la vie continue) et les sacs de voyage.

10 Sur les véhicules. On trouve la marque de la voiture, du vélo ou de la moto (14 items dont Suzuki, Peugeot, Nissan, etc.) mais aussi des termes divers (17 items) : noms de société (Brasseries du Cameroun), invocations (Grâce à Dieu tout est possible).

11 Sur des produits divers. 24 textes ont été lus sur des cartons et des sacs, 9 ont été lus sur la vaisselle, 8 sur des boîtes de conserve, 10 sur les montres, 10 sur des postes de radio, 28 sur des cassettes, 8 sur des ballons et 21 sur des cartables et sacs de voyage.

Ces textes indiquent soit l'origine des produits (Made in Nigeria sur la vaisselle, Cimencam sur un sac), soit le nom de la marque (sur les postes de radio, les emballages, les cahiers). Il est à noter que les produits de consommation courante sont peu variés et que les textes figurant sur l'emballage sont à peu près les mêmes qu'en Europe (marque, lieu de fabrication, composition etc.).

13 Sur les boissons, les cigarettes et les publicités correspondantes. 17 items ont pu être relevés sur des boissons et 9 sur des cigarettes. Il s'agit en général du nom du produit et éventuellement d'un slogan publicitaire. L'environnement publicitaire à Bibemi n'est cependant pas comparable à ce qu'on voit en Europe : les affiches vantant par exemple la bière sont petites et peu nombreuses, uniquement situées sur le mur de façade des deux bars.

14 Divers. Quelques textes ont été exceptionnellement relevés par les élèves sur d'autres supports : billets de banque, calendrier publicitaire (Sonel Toute notre énergie pour vous servir), inscription sur une porte (Malheur aux filous), sur un poteau électrique (Défense de toucher aux fils même tombés à terre), graffiti sur un arbre (Asta bordel et Jésus est mon sauveur), convocation chez le sous-préfet.

15 Si la plupart des élèves n'a pas prêté attention à ces textes, c'est certainement parce qu'ils sont plus rares ou moins accessibles (les enfants n'ont pas souvent, dans ce milieu, des 
billets de banque dans les mains; les calendriers publicitaires, en principe gratuits, s'achètent ou s'obtiennent par relations, etc...).

\subsection{La télévision}

La radio et la télévision émettent en français et en anglais, seulement après $18 \mathrm{~h}$. Peu d'élèves regardent la télévision (dans la classe, 40 élèves sur 81 affirment ne pas l'avoir regardée pendant la semaine ayant précédé l'enquête, 41 disent l'avoir regardée le weekend et seulement 4 le jour précédent). S'il y a peu de postes à Bibemi, il est habituel d'aller voir la télévision chez le voisin.

Les textes qu'on y voit sont les mêmes qu'en Europe: noms des produits, titre des émissions, listes des participants, etc.

\subsection{Les livres}

Les 79 élèves ayant participé à l'enquête peuvent être répartis en quatre groupes :

- 24 (soit le tiers environ) indiquent qu'ils n'ont lu aucun livre pendant le mois précédent et peuvent être considérés connue des non-lecteurs. Leur nombre est peut-être même plus important. Il est possible que certains élèves n'aient pas répondu sincèrement dans l'espoir d'obtenir une bonne note ;

- 14 élèves disent avoir lu un livre ou un fascicule distribué par les missions religieuses ( $L a$ Bible, Jésus guérit un lépreux etc.) ;

- 9 élèves disent avoir lu une bande dessinée (Lucky Luke) ou un magazine (Nous deux);

- 32 enfin affirment avoir lu un roman, un recueil de nouvelles ou une pièce de théâtre. Certains titres cités sont peu connus (on peut même imaginer que certains enfants confondent roman et revue) ; plusieurs - de façon inattendue car le collège ne comporte que des classes de $6^{\mathrm{e}}$ - citent des œuvres au programme des classes supérieures (Le Vieux Nègre et la Médaille et Trois prétendants, un mari, au programme des classes de $4{ }^{\mathrm{e}}$, Les Bimanes au programme des classes de $3^{\mathrm{e}}$ ). Quelques sondages ont montré que les livres cités avaient au moins en partie été effectivement lus.

\section{Bilan}

Les occasions de lire dans la rue sont rares. Les enfants de Bibemi sont très peu sollicités par l'écrit dans leur vie de tous les jours. Leur situation sur ce point n'est en rien comparable à celle des élèves européens.

La publicité est pratiquement inexistante. Il n'y a, dans la ville, ni librairie, ni marchand de journaux. L'information se fait par la radio et pas par les journaux ou revues exposés aux regards.

21 Caré et Debyser dans l'article "Lire la rue dans la rue ", ont dû se limiter à "cent (et quelques) mètres de rue» parisienne, en excluant "les affiches publicitaires et les devantures de marchands de journaux et de librairie». A Bibemi, par contre, il a été possible d'établir une liste apparemment exhaustive des textes inscrits sur les panneaux de la ville.

22 La plupart des textes que l'on trouve ont une fonction d'information (direction, nom d'un produit, usage d'un bâtiment) ou quelquefois d'avertissement (Malheur aux filous). 

(Camsuco, pur sucre de canne extra raffiné). Les phrases, même nominales, sont rares (Défense d'entrer aux étrangers ou alors Grâce à Dieu, tout est possible). Dans certains cas, les élèves n'ont pas tout noté sur leurs fiches. Par exemple sur une boîte de lait en poudre on peut lire le mode d'emploi, la composition et un texte d'une dizaine de lignes (en anglais et en français) vantant les qualités de ce lait "pur, délicieux, nourrissant ». Cette situation reste cependant limitée aux produits d'origine européenne. La lessive - importée en fraude du Nigéria - est, par exemple, vendue dans des sacs plastiques sans aucune inscription.

\section{l'êt} le et dans laquelle sont enseignées toutes les matières (Gérard Vigner propose le concept de langue d'information ou langue de scolarisation). Il est clair - et les enseignants de la discipline l'ont remarqué - que, pour des élèves qui ne connaissent pas vraiment la langue, la difficulté à résoudre certains problèmes de mathématiques tient plus à l'incompréhension du sujet qu'à un manque de logique.

\subsection{Perspectives}

27 Quel français connaîtront et parleront les enfants de Bibemi dans quelques années? On assiste actuellement à une scolarisation massive sans beaucoup de moyens en ce qui concerne l'enseignement du français, pour lequel il n'y a ni livres, ni manuels. Il est probable (si l'on en juge par ce qu'on lit, par exemple, dans les copies de BEPC) que beaucoup d'élèves ne maîtriseront jamais cette langue.

La situation de Bibemi n'est, de ce point de vue-là, en rien exceptionnelle. Toutes les souspréfectures et même les préfectures du Cameroun septentrional présentent un « environnement écrit » aussi réduit. Ce n'est que dans les chefs-lieux de province qu'on a plus d'occasions de lire, c'est-à-dire de se procurer des livres et de lire quelque chose sur un mur, sur un objet, etc. Il semble bien qu'on trouve la même situation dans certaines parties du Tchad, du Niger.

29 Les quelques tentatives de certains professeurs pour obliger les élèves à utiliser le français lorsqu'ils communiquent entre eux (par exemple dans la cour de récréation) ont été vaines. Il n'est, par ailleurs, guère possible d'accroître le nombre de textes offerts à la lecture en ville ou sur les produits. La seule action possible semble être actuellement la création de bibliothèques scolaires ou communales de façon à aider les élèves qui souhaitent lire (environ la moitié d'après l'enquête) à se procurer des livres; mais des 
difficultés apparaissent vite : disposer d'un local et d'une personne de confiance, obtenir les livres.

Une telle démarche ne résoudra cependant pas le problème de fond mis à jour par cette enquête : la difficulté des enfants à apprendre à lire dans un environnement n'offrant dans la vie quotidienne que très peu d'occasions de contacts avec les textes écrits.

\section{BIBLIOGRAPHIE}

CARE J.-M. \& DEBYSER F., « Lire la rue dans la rue », LeFrançais dans le Monde. n 141. pp. 52-59, 1979.

DUPONCHEL L., « Le français en Côte d'Ivoire, au Dahomey et au Togo », in Valdman Albert (dir.) : Le français hors de France, Champion, Paris. 1979.

LAFAGE S., Français écrit et parlé en pays éwé, SELAF, Paris, 1985.

VIGNER G., « Le français langue de scolarisation », Diagonales, n 12, pp. 41-45, 1989.

\section{ANNEXES}

\section{Annexes}

\section{Textes écrits sur des panneaux ou pancartes}

CES DE BIBEMI - POSTE DE SOUDURE - BIENVENUE À BIBEMI - HÔPITAL SILENCE PARKING HÔPITAL - ESCALE BAR - ADOUMRI PITOA GAROUA - CENTRAL BAR BIBEMI - 33 EXPORT - L'AMIE DES AMIS - POSTE SODECOTON - GENDARMERIE DE BIBEMI - SOUSPRÉFECTURE DE BIBEMI - CHEFFERIE BIBEMI - COMMISSARIAT SPECIAL DE BIBEMI MOULIN A.V.P. -ÉCOLE MATERNELLE, BP. 979, GAROUA - STATION ZOOTECHNIQUE ET VÉTERINAIRE DE BIBEMI, (peu lisible) - PADERME DJALOUMI - SODECOTON SECTEUR DE DÉVELOPPEMENT RURAL DE BIBEMI - DÉFENSE D'ENTRER AUX ÉTRANGERS.

MINISTÈRE DE L'ÉDUCATION NATIONALE - DÉLÉGATION PROVINCIALE DU NORD INSPECTION DÉPARTEMENTALE DE LA BENOUE - SOUS-INSPECTION DE L'ENSEIGNEMENT PRIMAIRE ET MATERNEL DE BIBEMI, BP. 979. GAROUA.

\section{Textes écrits sur des vêtements}

RDPC - UNDP - MDR - UPC - ALLEZ LES LIONS - ALLEZ LES LIONS INDOMPTABLES VERITABLE WAX - WAX DE LUXE - WAX ANGLAIS - GUARANTEED SUPER WAX - MOSQUÉE DE GAROUA - ADIDAS - JUMPING - LACOSTE - JOURNÉE INTERNATIONALE DE LA FEMME GROUPE DES FEMMES DU CAMEROUN - $1^{\text {ER }}$ MAI FÊTE DU TRAVAIL - RÉPUBLIQUE DU CAMEROUN - LA FOI D'UNE FEMME EN CHRIST - LOUONS LE SEIGNEUR - MÉDECINS SANS 
FRONTIÈRES - « 33 » EXPORT - GUINNESS IS GOOD FOR YOU - COCA COLA - SUPER PRINCE

- HUGO BOSS - MANU CYCLE - LAISSE-MOI LA VIE - LÈVE TOI ET BRILLE - TOUT PASSE MAIS LA VIE CONTINUE - NOUS DEUX JUSQU'À LA MORT - JE SAIS CE QUE JE VEUX DELTA - MONDIAL 90 - PATTAYA - COQ SPORTIF - JOYEUX NOËL.

\section{Textes écrits sur des véhicules}

BON VOYAGE - BONNE CHANCE - GRÂCE À DIEU TOUT EST POSSIBLE - I LOVE YOU - TAXI BRASSERIES DU CAMEROUN - MOBIL - AIR DZIBUILAO - AIR MOULVOUDAYE - AIGLE DU NORD - PROJET NEB - TRANSPORT INTERDIT - COMMUNE RURALE DE GAROUA L'ÉLÉPHANT DE LA PISTE - MALTA GUINNESS - DELTA - $60 \mathrm{KM} / \mathrm{H}$.

\section{RÉSUMÉS}

Dans la partie septentrionale du Cameroun (et peut-être dans d'autres états limitrophes), pour un enfant scolarisé en français les principales lectures sont celles de l'école (manuels, cahiers, tableau noir). Il a, la plupart du temps, peu d'occasions de lire ou bien les « écrits de la rue » ou bien des livres, magazines, etc.

In the northern regions of Cameroon (and perhaps in other bordering countries), the main reading opportunities, for a child whose schooling is conducted in French, are at school (schoolbooks, note-books, blackboard). Most of the time, he bas very few occasions to read, either notices in the streets, or else books, magazines, etc.

\section{INDEX}

Mots-clés : environnement écrit, langue de scolarisation, lire

Keywords : external environment, language of instruction, reading, written word

\section{AUTEUR}

\section{ANDRÉ CARRÉE}

Conseiller pédagogique, Gouara, Cameroun 